Revue internationale P.M.E.

Économie et gestion de la petite et moyenne entreprise

\title{
Une étude empirique des systèmes d'information marketing dans les PME manufacturières
}

\section{Louis Raymond, Jacques Brisoux et Abdellah Azami}

Volume 13, numéro 3-4, 2000

URI : https://id.erudit.org/iderudit/1008681ar

DOI : https://doi.org/10.7202/1008681ar

Aller au sommaire du numéro

Éditeur(s)

Presses de l'Université du Québec

ISSN

0776-5436 (imprimé)

1918-9699 (numérique)

Découvrir la revue

Citer cet article

Raymond, L., Brisoux, J. \& Azami, A. (2000). Une étude empirique des systèmes d'information marketing dans les PME manufacturières. Revue internationale P.M.E., 13(3-4), 63-87. https://doi.org/10.7202/1008681ar
Résumé de l'article

Une étude exploratoire réalisée auprès de 54 PME manufacturières québécoises a permis de décrire leurs pratiques en matière de système d'information marketing (SIMk), soit en ce qui a trait à la collecte d'information, à l'analyse et à la diffusion de cette information. L'analyse structurelle d'un modèle causal à l'aide de la technique PLS a aussi permis d'identifier certains déterminants et effets du SIMk, notamment son influence sur les décisions marketing et la performance des PME. 


\title{
Une étude empirique des systèmes d'information marketing dans les PME manufacturières
}

Louis RAYMOND

Jacques BRISOUX

Abdellah AZAMI

Université du Québec à Trois-Rivières

MOTS CLÉS

\section{Systèmes d'information - Technologies de l'information Marketing - Systèmes d'information marketing Décisions marketing - Marketing mix Performance PME manufacturières}

\begin{abstract}
RÉSUMÉ
Une étude exploratoire réalisée auprès de 54 PME manufacturières québécoises a permis de décrire leurs pratiques en matière de système d'information marketing (SIMk), soit en ce qui a trait à la collecte d'information, à l'analyse et à la diffusion
\end{abstract}

\section{LES AUTEURS}

Louis Raymond, Ph.D., est professeur titulaire de systèmes d'information à l'Université du Québec à Trois-Rivières et chercheur à l'Institut de recherche sur les PME. Ses recherches portent actuellement sur l'impact des technologies de l'information sur la performance des PME. Ses travaux ont été publiés dans des revues telles que MIS Quarterly, Entrepreneurship Theory and Practice, International Journal of Technology Management, International Journal of Electronic Commerce et Journal of Small Business Management. Adresse : 3351, boul. des Forges, C.P. 500, Trois-Rivières, Québec, Canada, G9A 5H7, téléphone: (819) 376-5080, courriel : < louis_raymond@uqtr.uquebec.ca >

Jacques Brisoux, Ph.D., est professeur titulaire de marketing à l'Université du Québec à Trois-Rivières. Membre de l'American Marketing Association (AMA) et de l'Association for Consumer Research (ACR), il est auteur ou coauteur de nombreuses publications dans le domaine du marketing et du comportement du consommateur. Depuis 1998, il est directeur de la coopération internationale à l'UQTR. Adresse: 3351 , boul. des Forges, C.P. 500, Trois-Rivières, Québec, G9A $5 \mathrm{H} 7$.

Abdellah Azami, M.Sc., est détenteur d'une maîtrise en gestion des PME et de leur environnement de l'Université du Québec à Trois-Rivières. II est agent de développement économique pour la Corporation de développement économique communautaire de Montréal. 
de cette information. L'analyse structurelle d'un modèle causal à l'aide de la technique PLS a aussi permis d'identifier certains déterminants et effets du SIMk, notamment son influence sur les décisions marketing et la performance des PME.

\begin{abstract}
An empirical study of 54 manufacturing SMEs allowed to describe their marketing information system (MkIS) practices, that is, in terms of data collection, analysis, and diffusion. Structural analysis of a causal model with the PLS technique also allowed us to identify certain determinants and effects of the MkIS, notably its influence on the SMEs' marketing decisions and performance.
\end{abstract}

\title{
RESUMEN
}

Una estudio empírica sobre 54 PyMEs manufactureras describe sus sistemas de información marketing (SIMK), sea las prácticas de colecta, análisis y difusión de informaciónes comerciales. Una análisis causal del modelo de investigación con la técnica PLS identifica ciertos determinantes y efectos del SIMk, especialmente su efecto sobre las decisiones marketing y los resultados de los PyMEs.

\section{ZUSAMMENFASSUNG}

Eine Forschungsstudie durchgeführt bei 54 kleinen und mittleren Unternehmen in Québec hat erlaubt, zu beschreiben, wie die Betriebe Marketinginformationssysteme (SIMk) aufbauen und anwenden. Sei es wie die Unternehmen Informationen holen, analysieren und die Informationen weiter verbreiten. Die strukturelle Analyse mit der Hilfe der Technik PLS hat erlaubt, gewisse Bestimmungsfaktoren und Effkete von Marketinginformationssystemen zu identifizieren. Vor allem sein Einfluss auf die Marketingentscheide und die Leistung der kleinen und mittleren Unternehmen.

\section{Introduction}

La capacité de survie des PME dépend de leur aptitude à prévoir les changements extérieurs et à s'adapter. Dans un environnement de plus en plus complexe et turbulent, les entreprises qui maintiennent et accroissent leur performance sont celles qui savent être à l'écoute du changement. Dans une PME, le dirigeant est censé être proactif et saisir toute information afin d'agir plus rapidement que ses concurrents et de bénéficier des occasions d'affaires du marché. Ce dernier doit mobiliser et motiver son équipe, fidéliser ses clients, identifier les besoins de ses prospects, surveiller ses concurrents, car ses principaux rivaux actuels ou à venir adoptent tous de nouvelles techniques, méthodes ou outils de gestion: systèmes d'information marketing, panels, sondages, bases de données et autres.

À cet égard, les systèmes d'information marketing (SIMk) sont censés jouer un rôle capital dans le succès d'une entreprise ; ils fournissent l'information dont l'entreprise a besoin pour une exploitation efficiente et une gestion efficace de sa 
fonction commerciale et pour obtenir ou maintenir son avantage concurrentiel (Samli, 1996). Dans le cas d'une PME, le SIMk peut souvent se réduire à des procédures de collecte d'information auprès des clients par les représentants ou d'analyse systématique et périodique de données secondaires publiées.

Le marketing a fait l'objet de rares investigations dans son application à la PME, que ce soit au niveau de l'emploi du «marketing mix» ou des activités spécifiques telles que la recherche marketing (Kinsey, 1987 ; Perreault, 1997). Le fait que ce domaine ait été négligé, tant par les chercheurs en marketing qu'en entrepreneuriat et PME, est d'autant plus important que les problèmes de marketing sont censés être très importants chez ces dernières (Huang et Brown, 1999) et en particulier depuis l'avènement de la mondialisation (Knight, 2000). Plus globalement, les quelques études empiriques portant sur la fonction marketing apportent peu de conclusions précises, en raison de l'application du concept vaste et multiforme du marketing à un univers d'une extrême hétérogénéité, la PME (Herrmann, 1993).

Devant l'hétérogénéité qui caractérise les PME, certains auteurs se sont interrogés sur l'existence de différences entre les pratiques marketing des PME (Herrmann, 1995). Ils ont aussi mis ces pratiques en relation avec plusieurs sources de diversité dans l'univers de la PME, comme le profil du dirigeant (Lorrain et Dussault, 1988), l'environnement de l'entreprise (Marchesnay, 1988) ou encore son stade de développement (Carson, 1985). Carson et Cromie (1990) retiennent que les caractéristiques des PME se traduisent sur le plan marketing par des ressources financières limitées, un manque d'expertise et un impact limité sur le marché. Les activités marketing semblent principalement menées en réaction à des crises opérationnelles, comme par exemple la perte d'un client important, dans le cas d'une entreprise de sous-traitance, ou la croissance insuffisante du chiffre d'affaires.

Cependant, l'importance des facteurs d'origine marketing pour le succès ou la survie des PME a été largement affirmée dans la littérature. À l'encontre de bien des préjugés, on peut affirmer qu'une stratégie marketing est encore plus nécessaire à la petite entreprise qu'à la grande entreprise, car elle permet de tracer une ligne de conduite dans un environnement difficile et changeant (Serraf, 1977, cité dans Herrmann, 1995). D'ailleurs, parmi les causes de mortalité et de défaillance des PME, l'absence d'une politique marketing est des plus fréquemment citées. Hills (1985) et Labarbera et Rosenberg (1989) ajoutent qu'en matière de création d'entreprises, un nombre élevé d'échecs s'explique par des erreurs d'appréciation du marché.

Devant ces constats, la présente étude porte sur les pratiques actuelles des PME manufacturières en matière de SIMk et sur l'impact de ces pratiques sur la prise de décision marketing et la performance de ces organisations. À titre exploratoire, il s'agira de tester empiriquement un modèle de recherche qui inclut certains déterminants présumés du SIMk, dont la stratégie marketing de la firme et les caractéristiques du responsable marketing. 


\section{Cadre conceptuel de recherche}

Les systèmes d'information marketing ont été définis comme l'ensemble des méthodes et des procédures servant à l'analyse, à la planification et à la présentation des informations nécessaires à la prise de décision en marketing (Assael, 1998). Le rôle des SIMk a d'abord été de fournir des rapports standardisés à de multiples utilisateurs en utilisant de nombreuses sources d'information (Choffray et Lillien, 1986). Aujourd'hui, les SIMk ne sont plus considérés uniquement comme des systèmes de gestion, mais également comme des systèmes opérationnels, principalement axés sur la gestion des activités de vente (Boulaire et Lefrançois, 1996).

Un SIMk n'est en fait qu'une approche structurée de recherche, de collecte, de traitement et d'entreposage de l'information dont le gestionnaire du marketing peut avoir besoin lors de ses prises de décision (Carrier, 1994). Dans la seule étude empirique traitant spécifiquement de SIMk en contexte de PME, Eldon (1997) indique que $51 \%$ des firmes interrogées disposent d'une certaine forme de système d'information marketing et que la plupart des responsables associent le SIMk aux rapports $(35 \%)$, aux bases de données et à la recherche documentaire $(18 \%)$.

Cependant, le SIMk peut être plus ou moins perfectionné et permettre à l'entreprise de recueillir un certain nombre de renseignements propres à orienter ses décisions. À cet effet, Higby et Farah (1991) avaient souligné l'utilisation des systèmes d'aide à la décision et des systèmes experts en marketing. Actuellement, des PME sont présentes sur l'Internet, ayant implanté un site sur le Web pour commercialiser et vendre leurs produits (Gallaugher et Auger, 1997). Néanmoins, et bien que le SIMk et la veille soient le plus souvent informatisés dans la grande entreprise, ce n'est pas toujours le cas dans les PME (Julien et al., 1999).

En l'absence d'un véritable courant de recherche et de modèles explicatifs des pratiques des PME en matière de SIMk, un modèle initial de recherche a été élaboré pour fins de validation empirique. Ce modèle, présenté à la figure 1 , est composé, d'une part, des quatre pratiques fondamentales en matière de SIMk (Assael, 1998), à savoir la collecte de l'information marketing, l'analyse et la diffusion de cette information ainsi que les technologies de l'information utilisées pour ce faire et, d'autre part, des décisions marketing que le SIMk est censé affecter. Les paragraphes suivants illustrent les résultats des quelques études empiriques qui ont examiné ces pratiques et ces décisions en contexte de PME.

\subsection{La collecte de l'information marketing dans les PME}

Comparativement à la grande entreprise, et en général, la PME ne dispose pas d'importantes sources d'information marketing (Eldon, 1997); elle dispose d'un réseau d'information et de contacts d'envergure restreinte, qui ne sont pas toujours suffisamment fiables pour être intégrés dans la stratégie de l'entreprise. À cet égard, 
FIGURE 1

Modèle de recherche

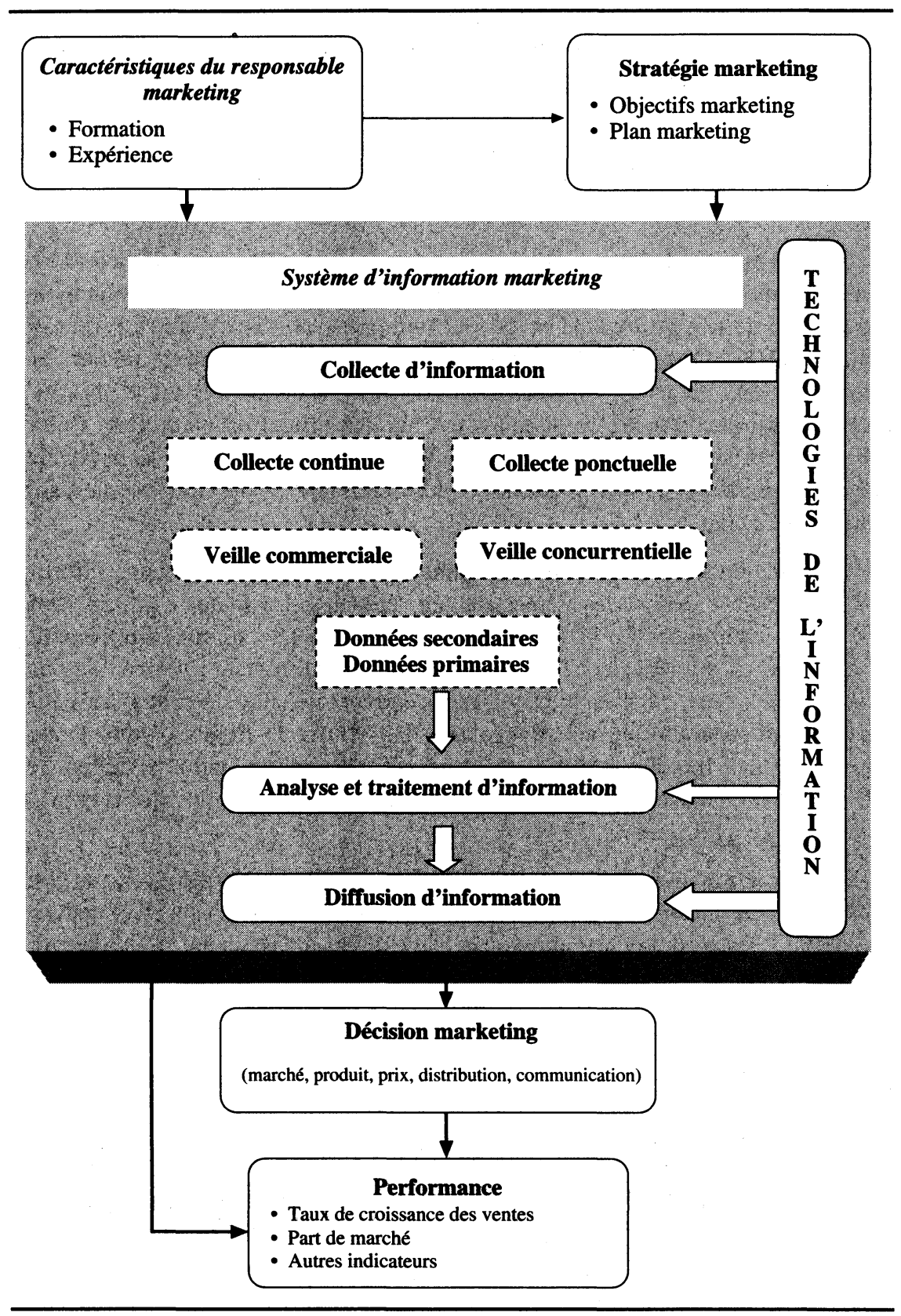


l'enquête de Johnson et Kuehn (1987) a montré que les dirigeants se fient davantage à leurs contacts personnels qu'aux sources économiques, gouvernementales ou technologiques; ils sont plus préoccupés par les informations externes. D'autres sources importantes sont les foires, les concurrents, ou encore d'autres intervenants de l'industrie (Lehtimaki, 1991). De plus, les vendeurs ou représentants sont en contact direct avec la clientèle de l'entreprise et, à ce titre, constituent des sources d'informations secondaires (rapports des ventes, etc.), voire primaires (participation à des groupes de discussion destinés à générer des idées de nouveaux produits, etc. ; Herrmann, 1995).

Dans son étude sur l'information portant sur les marchés, Bruch (1992) distingue quatre types de sources personnelles les plus utilisées: les clients, les contacts d'affaires, les experts et les fournisseurs. Quant aux sources écrites, les revues d'affaires sont les plus utilisées. El Louadi (1994) note le recours aux journaux et aux magazines spécialisés dans la quasi-totalité des cas. Concernant les informations disponibles pour le système d'information marketing, l'étude d'Eldon (1997) révèle que plusieurs PME dépendent de leurs données comptables, qui sont considérées comme la source la plus importante de données internes, ainsi que sur leurs clients.

Il convient cependant de ne pas oublier que les modes de collecte d'information de la PME ne sont pas les mêmes que ceux de la grande entreprise (Lesca, 1989). Les PME sont limitées par la difficulté à trouver de bonnes sources d'information et, par la suite, à utiliser cette information de façon efficace. Elles ont en outre beaucoup plus de difficultés que les grandes entreprises à obtenir cette information récente, souvent contrôlée ou partagée dans des groupes restreints, compte tenu de leurs ressources limitées et d'une structure nationale de production et de transfert de l'information mal adaptée ou mal orientée vers les besoins des petites entreprises (Jacob, Julien et Raymond, 1997).

Fann et Smeltzer (1989) rapportent que la majorité des dirigeants de PME obtiennent leur information d'une façon très informelle, en observant et en analysant les produits de leurs concurrents et en échangeant avec les clients et les vendeurs. Selon ces auteurs, les dirigeants de PME privilégient les communications orales directes et scrutent d'une façon routinière leurs clients et leurs concurrents.

\subsection{L'analyse, le traitement et les technologies de l'information}

Julien et al. (1999) soulignent le fait que, bien que les PME soient remarquablement actives dans leur recherche d'informations externes en utilisant une multitude de sources et de moyens, il leur manque encore la capacité informationnelle nécessaire pour traiter ces informations. Généralement, l'organisation de l'information est établie selon un mode qui la rend difficilement exploitable pour la prise de 
décision stratégique ; ainsi, l'information de fonctionnement prime largement sur l'information à caractère plus stratégique (Dubois, 1979). Outre sa rareté, l'information disponible sous une forme synthétique n'est pas interprétée rigoureusement ; le rôle central et l'implication du dirigeant dans toutes les décisions semblent être à l'origine d'une forte subjectivité. En effet, la capacité restreinte de contrôler l'environnement et d'interpréter les informations disponibles entraîne de fréquentes crises de décision (Fallery, 1983). Enfin, l'étude réalisée par Kinsey (1987) révèle le fait qu' un certain nombre de répondants avouent leur incapacité à donner un sens à l'information provenant du marché.

Dans un environnement de plus en plus complexe et incertain, la maîtrise des technologies de l'information (TI) est devenue l'un des facteurs critiques de succès pour les organisations (Raymond et Blili, 1997). L'impact des TI sur les entreprises est tel que leur manière de communiquer s'en trouve modifiée, qu'il s'agisse de communication interne au sein de l'entreprise ou de communication externe, entre l'entreprise et son environnement (Keen, 1991). Les TI contribuent à remodeler les bases de gestion des entreprises ; elles affectent le service à la clientèle, les opérations, la production et les stratégies marketing de communication et de distribution; elles permettent de sélectionner l'information, de l'interpréter et de l'analyser plus facilement et plus rapidement (Salern, 1997).

\subsection{La diffusion de l'information}

La structure moins formelle de la PME affecte la circulation de l'information. Les PME sont connues pour la prédominance de relations directes, interpersonnelles et orales en matière de circulation d'information (Herrmann, 1995). Dans ce sens, certains auteurs notent que s'il fallait définir la PME du point de vue de son système de relations sociales, il faudrait la définir comme un système de relations immédiates ou directes par opposition aux systèmes de relations sociales fonctionnelles et médiatisées de la grande entreprise (Wren, Simpson et Paul, 1998). Les discussions informelles constituent dans ce cas un moyen puissant de communication dans la PME (Jaworski et Kohli, 1993). Eldon (1997) souligne qu'une grande partie des PME interrogées n'utilisent pas de moyens informatiques tels qu'un Intranet pour communiquer entre les différents services et sections. Et peu nombreuses sont les PME qui utilisent le courrier électronique pour diffuser l'information.

\subsection{Les décisions marketing: le marketing mix}

Les décisions marketing se regroupent naturellement autour du choix des cibles de marché et du marketing mix (Saporta, 1986; Eldon, 1997). Contrairement à la grande entreprise qui peut opter pour une stratégie de marketing différencié (multisegments), la PME privilégie souvent une stratégie de marketing concentré 
sur un seul segment de marché (Brisoux, 1995). Le marketing mix est le dosage des différents efforts de marketing relatifs au produit, au prix, à la distribution et à la communication (Assael, 1998). Les actions menées dans ces quatre domaines peuvent évidemment présenter des aspects à la fois stratégiques et opérationnels.

\subsubsection{Les décisions relatives au produit}

C'est d'abord au niveau du produit que se situent probablement les points forts des PME (Herrmann, 1995). En matière de développement de nouveaux produits et dans le prolongement de ce qui a été écrit précédemment, il semble que les PME s'appuient sur leurs clients lorsqu'elles décident de développer de nouveaux produits (Herrmann, 1995). Johne et Rowntree (1991) concluent que la plupart des produits sont développés en relation avec des clients, soit du fait de l'incitation directe de quelques clients, soit à l'initiative de l'entreprise pour répondre aux besoins émergents d'un petit nombre de clients avec lesquels elle a toujours entretenu un contact étroit. Dans ce cas, il s'agit somme toute d'une orientation « ventes » plutôt que d'une orientation «marché».

\subsubsection{Les décisions relatives au prix}

Relativement à ce que font les PME en matière de détermination des prix, la plupart des auteurs (Kinsey, 1987 ; Ellis et Jolibert, 1991) concluent que les prix des produits paraissent plutôt fixés selon l'approche « coût plus marge ». Gardner (1983) précise tout de même que la moitié des entreprises interrogées tiennent nettement compte de leurs objectifs organisationnels en adoptant cette méthode. Ce dernier a constaté que seules deux entreprises sur les 15 étudiées appliquaient strictement l'approche «cost plus » pour fixer leurs prix de vente. Pour Carson et Cromie (1990) et pour Méziou (1991), la fixation des prix intègre les prix des concurrents ainsi que les perceptions et attitudes des clients.

Pour d'autres auteurs, le prix n'apparaît pas comme une « arme essentielle » (Herrmann, 1996; Marchesnay, 1988). Jackson, Douglass et Hertel (1979) soulignent, pour leur part, que $35 \%$ des petites entreprises interrogées déclarent s'aligner sur les prix des concurrents ou établissent des prix inférieurs. Conséquemment, le prix ne serait pas en général la variable faisant l'objet de la plus grande attention, celle-ci se portant plutôt sur le produit. Dans le cas de PME de haute technologie, les résultats de Traynor et Traynor (1989) soutiennent également cette observation. Le prix découlerait du résultat d'une négociation avec le client qui a certaines exigences concernant le produit, la connaissance des coûts de revient étant d'ailleurs peu probable initialement dans la PME (Marchesnay, 1988). 


\subsubsection{Les décisions relatives à la distribution}

La distribution des produits fabriqués par les PME est caractérisée par le recours à la vente directe (Herrmann, 1995); elle se résume à l'interaction personnelle du dirigeant avec le client. Dans le cas de très petites entreprises high-tech en phase de démarrage, Crick et Jones (2000) ont constaté que la stratégie d'innovation nécessite de suivre de près les besoins de la clientèle. Ces entreprises ressentent alors rapidement un besoin d'études de marché et surtout d'une force de vente. Dans les entreprises un peu moins jeunes, la plupart du temps, il n'existe pas de service commercial. Les dirigeants se déclarent cependant intéressés par le recrutement de représentants technico-commerciaux.

Les observations de Kinsey (1987) corroborent la forte implication personnelle du dirigeant dans les relations avec les clients; en fait, dans de nombreuses entreprises étudiées, le dirigeant constitue, à lui seul, la force de vente de l'entreprise. Et cette interaction du dirigeant avec la clientèle est souvent perçue, voire parfois présentée, comme garante d'une bonne connaissance du client et de ses besoins (Herrmann, 1995).

\subsubsection{Les décisions relatives à la communication}

Les auteurs n'hésitent pas à qualifier les actions de communication de quasi inexistantes en PME (Herrmann, 1995); la promotion se limite à une présence dans les salons, foires et autres expositions et à quelques plaquettes de présentation accompagnant cette démarche (Marchesnay, 1988). Les résultats de l'étude menée par Ellis et Jolibert (1991) révèlent que des PME survivantes diffèrent d'entreprises faillies dans l'utilisation d'une multiplicité de moyens de publicité et de promotion, incluant les dépliants, les catalogues et les présentations de la force de vente. Pour Gardner (1983), dans le cas des entreprises moyennes, la communication constitue la composante la plus faible de leur marketing mix et la plupart n'ont pas recours au service d'une agence de publicité, les supports de communication privilégiés demeurant le catalogue et les brochures publicitaires.

\subsection{Hypothèses de recherche}

Si l'on se reporte à la figure 1 , la sophistication du système d'information marketing est définie à travers les activités de collecte, d'analyse et de diffusion de l'information que réalise la PME. On peut déduire de la littérature en systèmes d'information et en marketing qu'un SIMk est censé exercer une influence non seulement sur les décisions marketing, mais aussi sur la performance de l'entreprise. On peut aussi déduire de cette même littérature et de celle en PME que la stratégie marketing ainsi que les caractéristiques du responsable marketing peuvent, à leur tour, exercer une influence sur les pratiques en matière de système d'information marketing. 
L'objectif premier de tout système d'information pour fins de gestion, incluant le SIMk, est de soutenir la prise de décision managériale et d'augmenter l'efficacité de l'organisation. À cet effet, un système dont la sophistication est plus élevée est censé produire une information de meilleure qualité (plus pertinente, plus opportune, plus complète, etc.) et contribuer à améliorer la performance (réduction des coûts, productivité, qualité du service, etc.), se reflétant ainsi dans les décisions prises à partir de cette information et dans l'augmentation des ventes ou des profits (Bergeron et Raymond, 1995). Les deux premières hypothèses de recherche sont donc les suivantes :

H1 : un système d'information marketing plus sophistiqué aura plus d'influence sur les décisions marketing de la PME;

$\mathrm{H} 2$ : un système d'information marketing plus sophistiqué aura plus d'influence sur la performance de la PME.

Par souci de cohérence du modèle de recherche avec l'importance démontrée du marketing mix dans la PME (Perreault, 1997), on devra évidemment postuler aussi une relation d'influence des décisions marketing sur la performance de l'entreprise.

Le comportement concurrentiel d'une entreprise, incluant ses activités de veille et de recherche marketing est orienté par sa stratégie marketing (Raymond, Julien et Ramangalahy, 2001 ; Varadarajan et Jayachandran, 1999). Ainsi, l'élaboration d'objectifs et la mise en place d'un plan marketing formel implique que la PME déploie les ressources informationnelles appropriées, c'est-à-dire qu'elle puisse vérifier l'atteinte de ses objectifs et la réalisation effective de son plan à l'aide de son SIMk. D'où la troisième hypothèse :

H3 : la PME dont la stratégie marketing est plus élaborée requiert un système d'information marketing plus sophistiqué.

Les caractéristiques individuelles sont cependant aussi déterminantes dans l'implantation et l'utilisation des systèmes d'information en PME, qu'elles soient centrées sur l'entrepreneur ou sur d'autres membres de la direction qui ont la responsabilité de ces systèmes (Thong, 1999). À cet effet, plusieurs études empiriques ont confirmé que des attributs tels que le niveau d'instruction et l'expérience ont une influence significative sur différents types de systèmes d'information tels les systèmes d'aide à la décision marketing et en PME (Zinkhan, Joachimsthaler et Kinnear, 1987 ; Raymond et Bergeron, 1992), d'où la quatrième hypothèse :

H4 : la PME dont le responsable marketing est plus éduqué et plus expérimenté aura un système d'information marketing plus sophistiqué. 
À nouveau, par souci de validité et de cohérence du modèle de recherche avec les résultats empiriques précédents sur le contexte de la planification marketing en PME (Carson et Cromie, 1990), on postulera une relation entre les caractéristiques du responsable marketing et le niveau d'élaboration de la stratégie marketing de la petite entreprise.

\section{Méthode de recherche}

Étant donné le nombre très limité d'études empiriques portant spécifiquement sur les systèmes d'information marketing en contexte de PME et le peu d'informations dont nous disposons sur les pratiques en cette matière par les dirigeants de ce type d'organisation, la nature de notre recherche est essentiellement descriptive et exploratoire. Un questionnaire d'enquête a été posté à un échantillon de $396 \mathrm{PME}$ manufacturières québécoises, sélectionnées aléatoirement parmi les entreprises ayant moins de 400 employés figurant dans la banque de données du Centre de recherche industrielle du Québec. Le répondant sollicité était l'individu responsable du marketing dans l'entreprise. Un total de 54 questionnaires dûment remplis par les responsables du marketing ont été retournés pour un taux de réponse de $14 \%$; étant donné la longueur du questionnaire et l'aversion des dirigeants de PME pour tout ce qui touche à la bureaucratie et à la paperasserie, ce taux de réponse est courant dans ce type d'enquêtes (Karimabady et Brunn, 1991).

Comme on le voit au tableau 1, les firmes échantillonnées proviennent de secteurs d'activité variés dont le bois, la fabrication d'équipements, le textile et autres. Elles ont en moyenne 100 employés, avec un minimum de 34 et un maximum de 350. Un peu plus du cinquième des répondants ont des responsabilités autres que la seule fonction marketing, ayant le titre de PDG, DG ou vice-PDG. L'expérience moyenne en tant que responsable du marketing est de 5 ans, avec un minimum inférieur à 1 an et un maximum de 30 ans.

Le questionnaire a été élaboré à partir des éléments du cadre conceptuel et d'instruments de mesure utilisés antérieurement. Les mesures des variables de sophistication du SIMk ont été adaptées des instruments de Bergeron et Raymond (1995) et de Julien et al. (1999). Une approche subjective plutôt qu'objective fut employée pour mesurer la performance des PME, étant donné que les mesures comptables sont souvent non disponibles ou non fiables dans ce contexte (Sapienza, Smith et Gannon, 1988). L'instrument utilisé provient de Venkatraman (1989); on y demande au répondant d'évaluer la position concurrentielle de sa firme en termes de croissance des ventes et de rentabilité. Compte tenu du contexte organisationnel moins complexe de la PME et des pratiques recensées dans la littérature citée plus haut, le niveau d'élaboration de la stratégie marketing fut mesurée par la présence d'objectifs marketing et d'un plan marketing formalisé. De même, les décisions marketing furent évaluées par la réalisation d'activités spécifiques censées en 
découler (p. ex., activités d'évaluation et de segmentation de marché pour les décisions marché). Les variables incluses dans le modèle de recherche et ainsi mesurées sont présentées au tableau 2, ainsi que leurs statistiques descriptives.

TABleaU 1

Secteurs d'activité des PME échantillonnées et titre des répondants $(n=54)$

\begin{tabular}{lllr}
\hline Secteurs d'activité & $\%$ & Titre du répondant & $\%$ \\
\hline Bois & 23 & Président-directeur général & 11 \\
Fabrication équipements & 19 & Directeur général & 6 \\
Textile & 13 & Vice-président-directeur général & 6 \\
Construction - acier & 13 & Directeur commercial & 43 \\
Alimentation & 11 & Vice-président ventes et marketing & 13 \\
Divers & 21 & Responsable marketing et communication & 21 \\
\hline
\end{tabular}

Les différentes relations postulées entre les construits de recherche ont été testées par l'entremise d'un modèle d'équations structurelles. La technique PLS (partial least squares) a été choisie parce qu'elle est plus appropriée en contexte de développement de théorie que la technique alternative LISREL (Fornell et Bookstein, 1982) plus connue. Contrairement à LISREL, PLS n'exige ni un échantillon de grande taille, ni des données dont la distribution est multinormale et accepte plus facilement des mesures ordinales ou dichotomiques (Fornell et Larcker, 1981).

\section{Résultats et discussion}

Les données obtenues nous permettent, d'une part, d'identifier les pratiques des PME en matière de systèmes d'information marketing et, $d$ 'autre part, d'identifier certains déterminants et impacts de ces systèmes sur la base du modèle de recherche proposé.

\subsection{Analyse descriptive}

On décrira ainsi le niveau de sophistication du SIMk des firmes échantillonnées ainsi que leurs décisions marketing.

Sources d'information. Trois fonctions semblent être les plus importantes sources d'informations internes selon les répondants : le marketing, qui est en contact direct avec le commercial, la direction générale (décisions et élaboration des plans) et, enfin, le commercial (selon les directives du marketing). Globalement, on peut dire que toutes les fonctions constituent une source d'information pour les PME dont l'importance varie selon les besoins de la firme. Concernant la fréquence d'utilisation des sources d'information, le personnel de vente, la direction et les collègues sont considérés comme les sources d'information internes les plus 
sollicitées, sans oublier le personnel de production ainsi que les bases de données internes. Les sources externes sont fréquemment utilisées ; à ce titre, les répondants semblent apprécier les clients comme la source d'information la plus utilisée, suivie des agents et représentants ainsi que des donneurs d'ordres.

Pour ce qui est des associations, les expositions et salons sont souvent sollicités, suivis des missions et voyages, ainsi que les associations sectorielles. Quant aux publications ou plus précisément, l'information écrite, les répondants identifient les revues spécialisées, les brochures et catalogues ainsi que les revues d'affaires comme les sources d'information écrites les plus utilisées. Quant aux moyens de collecte d'information, les contacts directs de personne à personne ainsi que les contacts téléphoniques sont les plus utilisés, sans oublier les réunions de travail informelles.

Recherche d'information sur les concurrents et les clients. Les clients constituent un domaine important pour la recherche d'information. Les répondants sont intéressés d'abord par les besoins de leurs clients ; ensuite, on trouve les critères de choix des clients ainsi que leur relation à long terme avec l'entreprise. L'élément concurrence constitue un point important pour les PME interrogées quant à la recherche d'information; près de la moitié des répondants déclarent surveiller souvent l'arrivée de nouveaux concurrents. Les forces et faiblesses des concurrents constituent un élément d'observation important dans un tiers des cas. Les répondants font souvent de la recherche d'information en ce qui concerne le prix des produits concurrents, leurs produits et services, les caractéristiques de leurs services ainsi que leur part de marché.

Analyse et traitement de l'information. L'activité d'analyse et de traitement d'information ne semble pas occuper une place importante dans les activités des PME interrogées. Les répondants déclarent procéder occasionnellement $(42 \%)$ à l'analyse et au traitement des informations marketing recueillies. Généralement, l'analyse se fait par des réunions de travail informelles (55\%) et formelles $(53 \%)$. Les études formelles et le traitement informatique ne semblent pas faire partie des moyens d'analyse et de traitement utilisés fréquemment par les PME interrogées. Les moyens techniques utilisés généralement dans cette activité sont les progiciels statistiques tels que SPSS et SAS (69\%), suivis des progiciels de bases de données et des tableurs tels que Access et Excel respectivement (31\%).

Diffusion de l'information. Seulement $21 \%$ des répondants confirment l'existence de règles formelles de diffusion de l'information marketing. Il semblerait que le moyen privilégié ou le plus utilisé pour la diffusion de l'information est le mode oral (contact direct de personne à personne : $70 \%$ ) suivi du support papier (distribution de rapports et mémos : $66 \%$ ). Quant au personnel impliqué dans le processus de collecte, d'analyse et de diffusion de l'information, il semblerait que le responsable commercial, les vendeurs et les représentants ainsi que le dirigeant sont très souvent impliqués dans ces activités. 
TABleAU 2

Statistiques descriptives des variables de recherche

\begin{tabular}{|c|c|c|c|}
\hline $\begin{array}{l}\text { Construit } \\
\text { Variable (nombre d'échelles) }\end{array}$ & Moy. & Min. & Max. \\
\hline \multicolumn{4}{|l|}{ Caractéristiques du responsable marketing } \\
\hline Expérience ${ }^{\mathrm{a}}$ & 5,4 & 0 & 30 \\
\hline Éducation ${ }^{\mathrm{b}}$ & 3,6 & 1 & 5 \\
\hline \multicolumn{4}{|l|}{ Élaboration de la stratégie marketing } \\
\hline Objectifs marketing ${ }^{\mathrm{c}}$ & 0,593 & 0 & 1 \\
\hline Plan marketing $\mathrm{c}$ & 0,722 & 0 & 1 \\
\hline Formalisation du plan ${ }^{c}$ & 0,481 & 0 & 1 \\
\hline \multicolumn{4}{|l|}{ Sophistication du système d'information marketing } \\
\hline Nombre de sources d'information (30) & 12,4 & 2 & 26 \\
\hline Nombre de moyens de collecte de l'information (12) & 5,6 & 0 & 12 \\
\hline Nombre de moyens d'analyse de l'information (5) & 2,4 & 0 & 4 \\
\hline Nombre de moyens de diffusion de l'information (4) & 2,0 & 0 & 3 \\
\hline Nombre de technologies de l'information (15) & 6,8 & 0 & 12 \\
\hline \multicolumn{4}{|l|}{ Déçisions marché } \\
\hline Évaluation du marchéc & 0,852 & 0 & 1 \\
\hline Segmentation du marchéc & 0,815 & 0 & 1 \\
\hline Positionnement $^{c}$ & 0,556 & 0 & 1 \\
\hline \multicolumn{4}{|l|}{ Décisions prix / produit } \\
\hline Nombre de critères de fixation de prix (5) & 2,2 & 1 & 5 \\
\hline Évaluation de la politique de prix ${ }^{c}$ & 0,944 & 0 & 1 \\
\hline Lancement de nouveaux produits ${ }^{c}$ & 0,667 & 0 & 1 \\
\hline Développement de nouveaux produits ${ }^{\mathrm{c}}$ & 0,944 & 0 & 1 \\
\hline \multicolumn{4}{|l|}{ Décisions distribution/communication } \\
\hline Circuit de distribution $^{\mathrm{d}}$ & 0,611 & 0 & 1 \\
\hline Évaluation de la politique de distribution ${ }^{c}$ & 0,685 & $\mathbf{0}$ & 1 \\
\hline Nombre de moyens de communication (15) & 3,7 & 0 & 10 \\
\hline Évaluation de la politique de communication ${ }^{c}$ & 0,537 & 0 & 1 \\
\hline \multicolumn{4}{|l|}{ Performance $^{e}$} \\
\hline Satisfaction du taux de croissance des ventes (1) & 3,9 & 2 & 5 \\
\hline Taux de croissance des ventes / concurrence (i) & 4,0 & 2 & 5 \\
\hline Taux de croissance de la part de marché / concurrence (1) & 3,8 & 2 & 5 \\
\hline Satisfaction du taux de profit sur l'actif de l'entreprise (1) & 3,3 & 1 & 5 \\
\hline Profits nets réalisés / concurrence (1) & 3,4 & 2 & 5 \\
\hline Taux de profit sur l'actif de l'entreprise / concurrence (1) & 3,5 & 2 & 5 \\
\hline Satisfaction de la marge de profit sur les ventes (1) & 3,4 & 1 & 5 \\
\hline Liquidité financière / concurrence (1) & 3,7 & 2 & 5 \\
\hline
\end{tabular}

a Nombre d'années en tant que responsable du marketing.

b Variable ordinale, 1 : secondaire, 2 : collège, $3:$ université ( $1^{\text {er }}$ cycle), $4:\left(2^{\mathrm{e}}\right), 5:\left(3^{\mathrm{e}}\right)$.

c Variables dichotomiques, 0 : non, 1 : oui.

d 0 : vente directe (de la firme aux clients), 1 : vente indirecte (par un distributeur).

e Échelles à 5 points, 1 : pas du tout satisfait, $2,3,4,5:$ très satisfait. 
Technologies de l'information. Nous avons aussi voulu connaitre la nature des technologies de l'information utilisées. Outre la présence de micro-ordinateurs dans plus des trois quarts des entreprises (77\%), on remarque la présence d'un lien Internet dans plus des deux tiers des cas $(70 \%)$. Les autres technologies présentes incluent les ordinateurs portatifs ( $68 \%$ ), le traitement de texte $(66 \%)$, la messagerie électronique (53\%), les réseaux locaux ( $42 \%)$, les bases de données $(40 \%)$, les mini-ordinateurs $(32 \%)$, les systèmes experts $(17 \%)$, la vidéoconférence $(6 \%)$ et les systèmes d'aide à la décision $(4 \%)$.

Décisions marketing-marché. Concernant l'évaluation du marché (analyse des tendances, etc.), les répondants déclarent procéder à cette activité, dans $87 \%$ des cas, et souvent, dans $49 \%$ des cas. En ce qui concerne la décision relative à la segmentation du marché, $83 \%$ des répondants déclarent procéder à cette activité et souvent, dans $43 \%$ des cas. Quant aux critères de segmentation, ce sont les critères géographiques et les avantages recherchés par les clients qui sont les plus utilisés. En ce qui a trait au positionnement, $65 \%$ des PME déclarent élaborer une stratégie de positionnement et $56 \%$ le font souvent.

Décisions marketing-produit. La même constatation a été faite au sujet de l'effort fourni pour développer et lancer de nouveaux produits. Nous avons remarqué une recherche constante d'une variété de produits offerts par les firmes que nous avons interrogées : $67 \%$ des PME procèdent au lancement de nouveaux produits et $94 \%$ procèdent au développement de nouveaux produits sur le marché (souvent dans $54 \%$ et $39 \%$ des cas respectivement).

Décision marketing-prix. L'ensemble des entreprises ont répondu qu'elles ont recours à une approche méthodique de fixation de prix. Quant aux méthodes utilisées pour fixer le prix, les répondants déclarent utiliser la technique de l'estimation des coûts $(89 \%)$ en se basant sur le prix de marché $(78 \%)$.

Décision marketing-distribution. L'analyse des résultats concernant la distribution démontre que les PME interrogées ont plus recours à la vente directe (67\%) qu'à la vente indirecte $(61 \%)$. En effet, la distribution des produits fabriqués par les PME, telle qu'elle a été étudiée par plusieurs auteurs, est caractérisée par le recours à la vente directe. Dans le cas de la vente indirecte, on remarque une dominance du canal court (fabricant, détaillant, consommateur).

Décision marketing-communication. Alors que l'ensemble des auteurs n'hésitent pas à qualifier les actions de communication de quasi inexistantes dans la PME, cela ne semble pas s'appliquer à notre échantillon, $89 \%$ des PME interrogées déclarant recourir à la communication pour promouvoir leurs produits et souvent, dans $45 \%$ des cas. La force de vente semble être le moyen privilégié par les PME. Ensuite, on trouve une multitude de moyens utilisés : la présence dans les salons, les brochures et les envois postaux, la promotion des ventes, les encarts dans certaines revues professionnelles, les commandites, les pages jaunes ainsi que 
les dépliants publicitaires. Contrairement à plusieurs études qui soulignent que les PME n'ont pas de budget de communication, $72 \%$ des PME interrogées déclarent en établir un. De plus, $67 \%$ des répondants déclarent procéder occasionnellement à cette activité. Le budget moyen alloué à la promotion et à la publicité représente en moyenne $2,1 \%$ du chiffre d'affaires.

\subsection{Analyse du modèle de mesure}

Conformément à la démarche de modélisation par équations structurelles, les tableaux 3 et 4 présentent les différents indices qui permettent d'indiquer la validité des construits utilisés dans le modèle de recherche. Les propriétés du modèle à être examinées sont la fidélité et la validité discriminante des construits du modèle proposé.

La fidélité d'une variable est mesurée par sa consistance interne à partir du coefficient rho, soit le rapport de la variance du construit sur la somme de cette même variance et de la variance d'erreur (Fornell et Larcker, 1981). Une valeur de rho supérieure à 0,60 indique que la variance du construit explique au moins $60 \%$ de la variance de mesure, ce qui est le cas pour tous les construits du modèle (tableau 3). La validité discriminante, c'est-à-dire à quel point les construits théoriques diffèrent les uns des autres ou représentent chacun un concept distinct, est évaluée à partir de la variance partagée (corrélation au carré) entre chaque paire de construits, qui devrait être inférieure à la variance moyenne extraite (VME) par le construit des indicateurs qui lui sont associés. Nous pouvons constater d'après le tableau 4 que ce critère est rencontré pour tous les construits du modèle.

Notons que, à la suite d'une analyse initiale, certaines variables ont été écartées car elles n'étaient pas saturées au niveau 0,5 sur le construit à mesurer. On ne retrouve donc au tableau 3 que les différentes variables retenues pour chaque construit.

\subsection{Analyse du modèle causal}

Les relations entre les construits sont validées en examinant le signe, la valeur et le seuil de signification des coefficients de causalité calculés par la méthode PLS, comme on peut le voir à la figure 2 .

Relation entre le SIMk et les décisions marketing (HI). Le traitement initial des données à l'aide de PLS a révélé que les décisions marketing, telles qu'elles ont été mesurées, ne constituaient pas un seul construit mais plutôt deux construits distincts, à savoir les décisions marché et les décisions prix/produit (les saturations des variables de décisions distribution / communication étant inférieures à 0,5 ). Le système d'information marketing a effectivement une influence positive et très significative sur les décisions de marché (l'évaluation du marché, la segmentation et le positionnement) avec un coefficient de causalité élevé $(0,588)$ et un seuil de 
TABleaU 3

Résultats de l'estimation du modèle de mesure (PLS)

\begin{tabular}{|c|c|c|c|}
\hline $\begin{array}{c}\text { Construit } \\
\text { Variable }\end{array}$ & $\lambda^{\mathbf{a}}$ & $\rho^{\mathbf{b}}$ & VME $^{c}$ \\
\hline $\begin{array}{l}\text { Caractéristiques du responsable marketing } \\
\text { Expérience dans le poste de responsable marketing } \\
\text { Éducation (retirée) }\end{array}$ & $\begin{array}{c}1,00 \\
-\end{array}$ & 1,00 & 1,00 \\
\hline $\begin{array}{l}\text { Elaboration de la stratégie marketing } \\
\text { Plan marketing } \\
\text { Formalisation du plan }\end{array}$ & $\begin{array}{l}0,75 \\
0,98\end{array}$ & 0,86 & 0,76 \\
\hline $\begin{array}{l}\text { Sophistication du système d'information marketing } \\
\text { Moyens de collecte d'information } \\
\text { Moyens d'analyse d'information } \\
\text { Moyens de diffusion d'information } \\
\text { Technologies de l'information }\end{array}$ & $\begin{array}{l}0,66 \\
0,75 \\
0,63 \\
0,82\end{array}$ & 0,81 & 0,52 \\
\hline $\begin{array}{l}\text { Décisions marché } \\
\text { Évaluation du marché } \\
\text { Segmentation du marché } \\
\text { Positionnement }\end{array}$ & $\begin{array}{l}0,77 \\
0,61 \\
0,88\end{array}$ & 0,71 & 0,57 \\
\hline $\begin{array}{l}\text { Décisions prix/produit } \\
\text { Critères de fixation de prix } \\
\text { Développement de nouveaux produits }\end{array}$ & $\begin{array}{l}0,56 \\
0,86\end{array}$ & 0,68 & 0,52 \\
\hline $\begin{array}{l}\text { Performance } \\
\text { Satisfaction du taux de croissance des ventes } \\
\text { Taux de croissance des ventes / concurrence } \\
\text { Taux de croissance de la part de marché / concurrence } \\
\text { Satisfaction du taux de profit sur l'actif de l'entreprise } \\
\text { Profits nets réalisés / concurrence } \\
\text { Taux de profit sur l'actif de l'entreprise / concurrence } \\
\text { Satisfaction de la marge de profit sur les ventes } \\
\text { Liquidité financière / concurrence }\end{array}$ & $\begin{array}{l}0,78 \\
0,81 \\
0,83 \\
0,74 \\
0,55 \\
0,56 \\
0,60 \\
0,59\end{array}$ & 0,84 & 0,48 \\
\hline
\end{tabular}

a Saturation de la variable sur le construit auquel elle est associée.

b Rho de Fornell et Larcker (coefficient de fidélité) $=(\hat{\mathrm{A}} \mathrm{l})^{2} /(\hat{\mathrm{A}} \mathrm{l})^{2}+\hat{\mathrm{A}}\left(1-\mathrm{1}^{2}\right)$.

c Variance moyenne extraite $=\left(\hat{\mathrm{A}} \mathrm{l}^{2}\right) / n$.

TABLEAU 4

Corrélations entre les construits du modèle de recherche (validité discriminante)

\begin{tabular}{lrccccr}
\hline & $\mathbf{1}$ & $\mathbf{2}$ & $\mathbf{3}$ & $\mathbf{4}$ & $\mathbf{5}$ & $\mathbf{6}$ \\
\hline 1. Expérience du responsable & 1,00 & & & & & \\
2. Stratégie marketing & $-0,40$ & 0,87 & & & & \\
3. Système d'information marketing & $-0,26$ & 0,40 & 0,72 & & & \\
4. Décisions marché & $-0,02$ & 0,18 & 0,56 & 0,75 & & \\
5. Décisions prix / produit & $-0,05$ & 0,09 & 0,21 & 0,23 & 0,72 & \\
6. Performance & $-0,05$ & 0,01 & 0,15 & 0,11 & 0,28 & 0,69 \\
\hline
\end{tabular}

a Diagonale : racine carrée de la variance moyenne extraite du construit $=(\mathrm{VME})^{1 / 2}$; sous-diagonale : corrélation entre les variables latentes $=(\text { variance partagée })^{1 / 2}$. 
FIGURE 2

Résultats de l'analyse avec PLS $(n=54)$

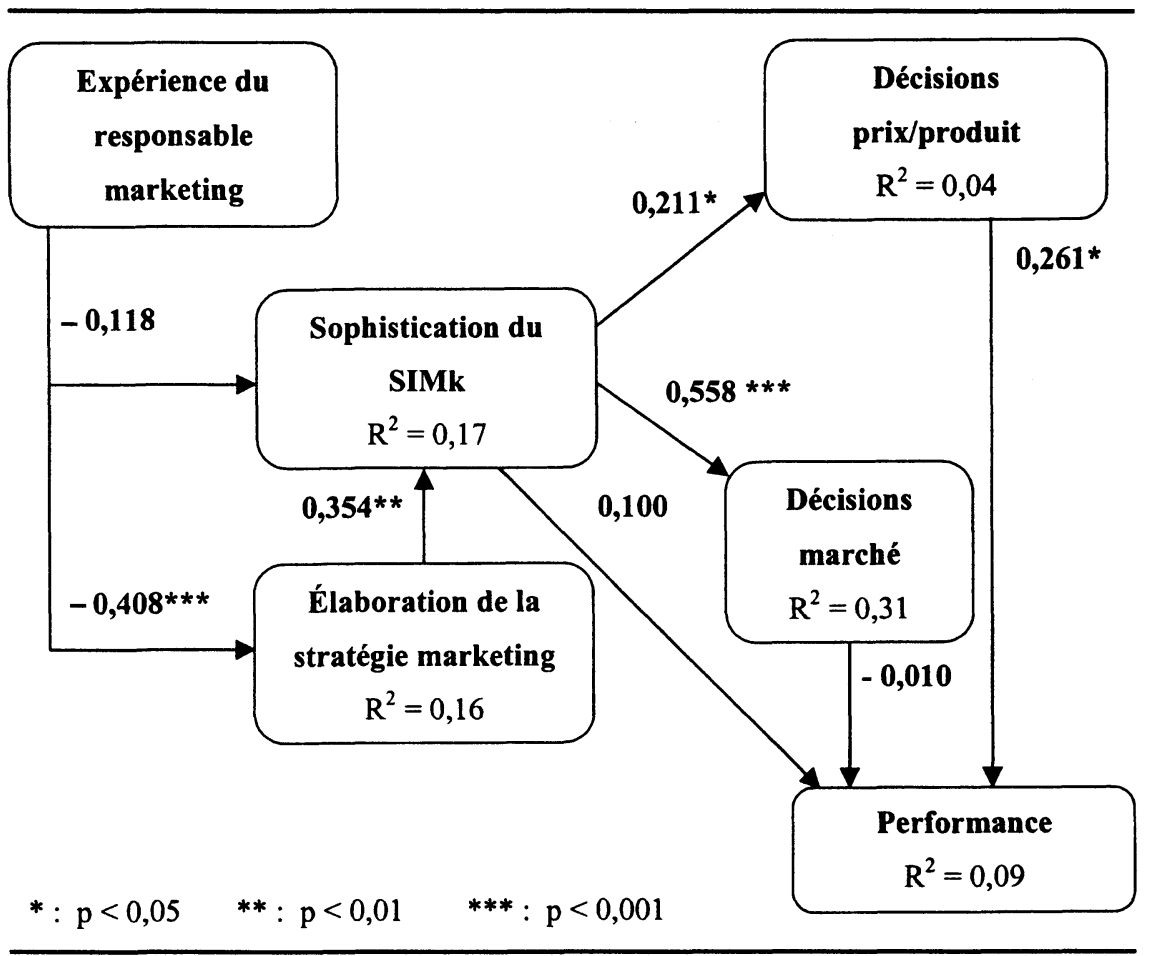

signification inférieur à 0,001 . En plus de soutenir les décisions relatives au marché, le système d'information marketing a aussi une influence positive sur les décisions de prix / produit, avec un coefficient de causalité de 0,211 et un seuil de signification inférieur à 0,005 . Cela voudrait dire que, dans le cas des décisions de développement de nouveaux produits et de fixation de prix, le responsable marketing requiert plus de moyens de collecte, d'analyse et de diffusion d'information, donc un SIMk plus sophistiqué.

Relation entre les décisions marketing et la performance. On peut constater que les décisions relatives au développement de nouveaux produits ainsi que celles relatives à la fixation du prix ont une influence directe sur la performance de l'entreprise. Le seuil de signification est inférieur à 0,05 pour un coefficient de causalité de 0,261 . Ce qui veut dire que l'entreprise qui développe de nouveaux produits et utilise plus de critères pour fixer le prix de ses produits aura une meilleure performance. 
Relation entre le SIMk et la performance (H2). Il semblerait que le système d'information marketing n'a pas d'influence directe sur la performance de l'entreprise. Compte tenu des résultats précédents, cette influence serait plutôt indirecte, c'est-à-dire qu'elle agirait par l'intermédiaire des décisions sur les prix et les produits.

Relation entre la stratégie marketing et le SIMk (H3). Les résultats de l'analyse nous révèlent que la stratégie marketing ou, plus précisément, le plan marketing a effectivement un effet positif sur la sophistication du SIMk. Le coefficient de causalité est de 0,354 avec un seuil de signification inférieur à 0,01. Cela veut dire que le SIMk est affecté directement par l'existence d'un plan marketing et par sa formalisation. Dans ce cas, on peut dire que lorsque le responsable marketing élabore un plan marketing formel, il aura besoin de moyens plus élaborés pour atteindre les objectifs de ce plan, et donc d'un système d'information marketing plus sophistiqué.

Relation entre le responsable marketing et le SIM $k(H 4)$. Il s'agit de vérifier si certaines caractéristiques du responsable marketing ont une influence sur le système d'information marketing. L'analyse causale n'a pu identifier de relation significative entre le niveau d'instruction et d'expérience du responsable marketing et la sophistication du SIMk.

Relation entre le responsable marketing et la stratégie marketing. Il y a effectivement une relation entre les caractéristiques du responsable marketing et la stratégie marketing, avec un coefficient de causalité négatif de $-0,408$ et un seuil de signification inférieur à 0,001 . Cela veut dire que plus le responsable marketing a de l'expérience dans le poste, moins il effectue de planification marketing et moins cette planification est formalisée. On peut interpréter ce résultat en remarquant que, dans la PME, l'expérience du dirigeant est souvent synonyme de savoir-faire, de jugement et d'intuition qui peuvent remplacer les méthodes et les techniques formelles de planification, de contrôle et de management (Carson et Cromie, 1990).

Globalement, ces résultats constituent donc une validation empirique partielle du modèle de recherche. Le résultat le plus saillant est qu'un système d'information marketing plus sophistiqué est associé d'abord et avant tout aux décisions de marché. Comme le marché représente la source de survie de toute entreprise, il est très plausible que toutes les décisions s'y rapportant bénéficient de la plus grande attention de la part des dirigeants de l'entreprise. Le responsable marketing d'une PME qui veut évaluer, segmenter le marché et positionner l'entreprise sera plus apte à le faire s'il bénéficie de sources d'information (représentants, clients, centres de recherche, etc.) et de moyens de collecte formels et informels plus diversifiés (contacts, recherches documentaires, études de marché, etc.) lui permettant, entre autres, de capter des « signaux faibles » dans l'environnement et de réagir ainsi plus rapidement aux nouvelles tendances du marché (veille commerciale). L'envergure 
et la richesse de l'information ainsi captée impliquent une analyse et un traitement plus approfondis, ainsi qu'une plus large diffusion de cette information aux acteurs concernés dans l'entreprise.

L'argumentation précédente peut tout aussi bien s'appliquer aux décisions marketing relatives au produit et au prix. L'entreprise qui veut développer et lancer de nouveaux produits en réponse aux tendances du marché qu'elle a décelées et qui veut établir des prix compétitifs pour ses produits à partir de critères plus appropriés que simplement les coûts de fabrication aura besoin d'information sur la concurrence et la technologie. Le SIMk devra ainsi fournir de l'information sur les procédés de fabrication existants et émergents, et sur les produits et les prix de ses compétiteurs (veille concurrentielle et technologique) et permettre l'analyse, le traitement et la diffusion de cette information pour déterminer les attributs du produit et son prix éventuel. Cela est d'autant plus important que ce sont ces décisions qui affectent ici directement la performance de la PME manufacturière.

Un autre résultat saillant de cette recherche est la confirmation d'une relation entre l'élaboration de la stratégie marketing de la PME manufacturière et le niveau de sophistication de son SIMk. Dans la mesure où l'entreprise formalise un plan marketing dans une démarche relativement formelle, elle aura tendance à se doter de mécanismes de pilotage dont, au premier chef, un système d'information permettant d'évaluer les résultats de la mise en œuvre du plan. À titre d'exemple, une sophistication accrue du SIMk se manifestera par la collecte et l'analyse de données relatives à la satisfaction de la clientèle et à la qualité des produits et services offerts par l'entreprise.

Finalement, l'impact des caractéristiques individuelles, plus précisément l'expérience du responsable marketing, ne se reflète sur la sophistication du SIMk qu'indirectement, soit par l'intermédiaire de la stratégie marketing. Dans la mesure où un dirigeant plus expérimenté a moins recours à une planification marketing formelle, ses besoins informationnels seraient moins explicites et demanderaient alors moins d'un système d'information.

\section{Limites et conclusion}

Cette étude a permis d'apporter un éclairage sur les façons de faire des PME en matière de système d'information marketing. Cependant, comme toute recherche, l'étude réalisée a des limites d'ordre conceptuel et méthodologique dont il faut tenir compte dans la portée des résultats. Ainsi, par souci de clarté et de parcimonie, un certain nombre de facteurs de contingence n'ont pas été pris en considération dans la conceptualisation du modèle de recherche. À titre d'exemples, mentionnons l'incertitude liée à l'environnement spécifique de l'entreprise qui peut avoir influencé ses comportements en matière de SIMk. Par ailleurs, les composantes de chacun 
des construits, notamment les décisions marketing, ne sont pas exhaustives. Outre les caractéristiques du responsable marketing et la stratégie marketing, d'autres facteurs tels que la dépendance commerciale de la PME manufacturière envers de grands donneurs d'ordres peuvent influencer ses pratiques en matière de SIMk. Sur le plan méthodologique, la nature et la taille de l'échantillon ne permettent pas de généraliser les résultats observés à l'ensemble des PME. Ces résultats ne reflètent que la perception des responsables marketing des entreprises. Par ailleurs, compte tenu de la nature exploratoire de l'étude, les mesures des différentes variables de la recherche n'ont pas fait l'objet d'une appréciation formelle de fidélité et de validité.

Il nous paraît néanmoins légitime de dégager certaines retombées de cette recherche à l'égard des chercheurs et des dirigeants. Les résultats obtenus quant à la nature et à l'impact des systèmes d'information marketing nous poussent à affirmer que ces systèmes ne devraient plus être négligés par les chercheurs en tant qu'objets d'étude, d' autant plus que le nouvel environnement d'affaires, caractérisé par la mondialisation des marchés, accroît leur importance pour les PME. Pour des recherches futures, il serait pertinent d'approfondir la définition des pratiques en matière de SIMk et de tenir compte de facteurs additionnels, en utilisant possiblement une approche contingente où l'impact sur la performance individuelle et organisationnelle dépendrait de la congruence (le «fit») entre l'environnement, la stratégie et le système d'information marketing. Il conviendrait également d'utiliser des approches interprétatives et des méthodes qualitatives afin de mieux comprendre les comportements des acteurs concernés par le développement et l'utilisation des SIMk dans les PME. Il semble aussi que nos résultats invitent les dirigeants de PME à se préoccuper de façon plus explicite du développement de leur système d'information marketing et du fonctionnement des processus qui y sont intégrés tels que la veille commerciale et concurrentielle. D'une part, il s'agirait d'aligner le développement du SIMk sur la stratégie marketing de l'entreprise et, d'autre part, de réaliser que la prise de décision marketing dépend d'abord et avant tout de la qualité de l'information disponible, et donc du système d'information qui la sous-tend.

\section{Bibliographie}

Assael, H. (1998), Marketing, Fort Worth, Texas, Dryden Press.

BELICH, T.J. et A.J. DUBINSKY (1999), «Information processing among exporters : an examination of small firms », Journal of Marketing Theory and Practice, vol. 7, $\mathrm{n}^{\circ} 4$, p. 45-58.

BERGERON, F. et L. RAYMOND (1995), "The contribution of IT to the bottom line : a contingency perspective of strategic dimensions », Proceedings of the 16th International Conference on Information Systems, Amsterdam, p. 167-181. 
BOUlAIRE, C. et P. LEFRANÇOIS (1996), « Systèmes d'information marketing, organisation imaginaire et post-modernité », Rapport de recherche, Université Laval, Centre de service d'orientation et de recherche sur la compétitivité internationale et l'ingénierie de l'entreprise réseau.

BRISOUX, J.E. (1995), «Image et positionnement des produits de la PME : une approche basée sur la catégorisation des marques », Présentation au Colloque international «La recherche au service des PME », Institut national des sciences comptables et de l'administration d'entreprises, Antananarivo, Madagascar, 29-31 mai.

BRUCH, C.G. (1992), « Marketplace information scanning activities of new manufacturing ventures », Journal of Small Business Management, vol. 30, $\mathrm{n}^{\circ}$ 4, p. 41-53.

CARRIER, S. (1994), Le marketing et la PME: l'option gagnante, Charlesbourg, Québec, Les Éditions Transcontinentales.

CARSON, D.J. (1985), «The evolution of information in small firms », European Journal of Marketing, vol. 19, $\mathrm{n}^{\circ}$ 5, p. 7-16.

CARSON, D.J. et S. CROMIE (1990), «Marketing planning in small entreprises : a model and some empirical evidence », The Journal of Consumer Marketing, vol. 7, été, p. 5-18.

ChOFFRAY, J.M. et G.L. LILliEN (1986), «A decision support system for evaluating sales prospects and launch strategies for new products ", Industrial Marketing Management, vol. 15, p. 75-95.

CRICK, D. et M.V. JONES (2000), «Small high-technology firms and international hightechnology markets », Journal of International Marketing, vol. 8, no 2, p. 63-85.

DoutriauX, J. et J.D. FerRAND (1995), «Technologies de l'information et performance des PME de conseil », Document de travail 95-70, Université d'Ottawa, octobre.

DuBoIS, P.L. (1979), « Marketing et création d'entreprise », Revue Française de Gestion, $\mathrm{n}^{\circ}$ 20, mars-avril, p. 45-49.

EL LOUADI, M. (1994), «Les besoins informationnels et les technologies de l'information dans les PME québécoises », Cahier de recherche DAE-08, Université du Québec à Trois-Rivières, Département d'administration et d'économie.

ELDON, Y.L.I. (1997), « Marketing information systems in small companies », Information Resources Management Journal, hiver, p. 27-35.

ELLIS D.S. et A.J.P. JOLIBERT (1991), «The role of marketing in the survival of small industrial firms in a developing region », dans G.E. Hills et R.W. Laforge, Research at the Marketing / Entrepreneurship Interface, Proceedings of the University of Illinois at Chicago Symposium on Marketing and Entrepreneurship, p. 126.

FALLERY, B. (1983), «Un système d'information pour les PME », Revue Française de Gestion, novembre-décembre, $\mathrm{n}^{\circ} 43$, p. 71-77.

FANN, G.L. et L. SMELTZER (1989), « The use of information from and about competitors in small business management », Entrepreneurship : Theory and Practice, été, p. 35-45.

FORNELL, C.R. et F.L. BOOKSTEIN (1982), « Two structural equation models : LISREL and PLS applied to consumer exit-voice theory », Journal of Marketing Research, vol. 19, p. $440-452$. 
FORNELL, C.R. et D.F. LARCKER (1981), «Two structural equation models with unobservable variables and measurement error », Journal of Marketing Research, vol. 18 , p. 39-50.

GALlAUGHER, J. et P. AUGER (1997), «Factors affecting the adoption of an Internet-based sales presence for small businesses ", The Information Society, vol. 13, $\mathrm{n}^{\circ} 4, \mathrm{p} .55-74$.

GARDNER, D.M. (1983), «The marketing concept : its dimensions for the "big" small firms », dans G.E. Hills, D.J. Barbary et L.R. Duffus, Marketing and Small Business / Entrepreneurship : Conceptual and Research Directions, Université de l'Illinois, Chicago, p. 51-62.

HART, S. et N. TZOKAS (1999), « The impact of marketing research activity on SME export performance : evidence from the UK», Journal of Small Business Management, vol. $37, \mathrm{n}^{\circ} 2$, p. 63-75.

HERRMANN, J.-L. (1993), « Le marketing en PME : point sur les recherches et repères pour la recherche », Communication au $\mathrm{IX}^{\mathrm{e}}$ Congrès international de l'Association française du marketing, ICN, Université de Nancy II.

HERRMANN, J.-L. (1995), Contribution à la connaissance de la mise en ceuvre du concept marketing en PME manufacturières : le cas du développement de produits nouveaux, thèse de doctorat ès sciences de gestion.

HERRMANN, J.-L. (1996), «L'applicabilité du concept marketing en PME manufacturières : mythe ou réalité ? », dans Actes du III Congrès international francophone de la PME, tome 2, Trois-Rivières, Québec, p. 1048-1068.

HIGBY, M.A. et B.N. FARAH (1991), «The status of marketing information systems, decision support systems and expert systems in the marketing function of US firms », Information Management, vol. 20, $\mathrm{n}^{\circ}$ 1, p. 29-35.

HILLS, G.E. (1985), « Market analysis in the business plan : venture capitalists' perceptions », Journal of Small Business Management, janvier, p. 38-46.

HILLS, G.E. (1987), « Marketing and entrepreneurship research issues : Scholarly justification », dans G.E. Hills, R.W. Laforge, Research at the Marketing / Entrepreneurship Interface, Proceedings of the University of Illinois at Chicago Symposium on Marketing and Entrepreneurship, p. 3-15.

HUANG, X. et A. BROWN (1999), «An analysis and classification of problems of small business », International Small Business Journal, vol. 18, $\mathrm{n}^{\circ} 1$, p. 73-85.

JACKSON, J.H., D.K. HAWES et F.M. HERTEL (1979), « Pricing and advertising practices in small retail businesses », American Journal of Small Business, vol. 4, $\mathrm{n}^{\circ} 2$, octobre, p. 22-34.

JACOB, R., P.-A. JULIEN et L. RAYMOND (1997), « Compétitivité, savoirs stratégiques et innovation: les leviers de l'apprentissage collectif en contexte de réseau », Revue internationale de gestion, vol. 22, $\mathrm{n}^{\circ}$ 3, p. 93-100.

JAWORSKI, B.J. et A.K. KOHLI (1993), « Market orientation : antecedents and consequences », Journal of Marketing, vol. 57, $\mathrm{n}^{\mathrm{0}}$ 3, p. 53-70.

JOHNE, A. et S. RowNTREE (1991), « High technology product development in small firms : a challenge for marketing specialists », Technovation, $n^{\circ} 4$, p. 247-259. 
JOHNSON, J.L. et R. KUEHN (1987), «The small business owner/manager's search for external information », Journal of Small Business Management, juillet, p. 53-60.

JULIEN, P.-A., L. RAYMOND, R. JACOB et C. RAMANGALAHY (1999), « Types of technological scanning in manufacturing SMEs: an empirical analysis of patterns and determinants », Entrepreneurship and Regional Development, vol. 11, p. 281-300.

KARIMABADY, H. et P.J. BRUNN (1991), «Postal surveys to small manufacturers », Industrial Marketing Management, vol. 45, $\mathrm{n}^{\circ}$ 4, p. 319-326.

KEEN, P. (1991), Shaping the Future Business Design Through Information Technology, Boston, Massachusetts, Harvard Business School Press.

KINSEY, J. (1987), « Marketing and the small manufacturing firm in Scotland : findings in a pilot survey », Journal of Small Business Management, vol. 25, $\mathrm{n}^{\circ}$ 2, p. 18-25.

KNIGHT, G. (2000), «Entrepreneurship and marketing strategy: the SME under globalization », Journal of International Marketing, vol. 8, $\mathrm{n}^{\circ} 2$, p. 12-32.

LABARbera, P.A. et S.A. Rosenberg (1989), «Marketing research and small entrepreneurial entreprises », Proceedings of the Research Symposium on the Marketing / Entrepreneurship Interface, The University of Illinois at Chicago.

LEHTIMAKI, A. (1991), «Management of the innovation process in small companies in Finland», IEEE Transactions on Engineering Management, vol. $38, \mathrm{n}^{\circ} 2$, mai, p. $120-126$.

LESCA, H. (1989), Information et adaptation de l'entreprise, Paris, Éditions Masson.

LORRAIN, J. et L. DUSSAULT (1988), «Les entrepreneurs artisans et opportunistes : une comparaison de leurs comportements de gestion », Revue internationale PME, vol. $1, \mathrm{n}^{\mathrm{o}} 2$, p. $157-176$.

MARCHESNAY, M. (1988), «La mercatique de la petite entreprise », Revue internationale $P M E$, vol. $1, \mathrm{n}^{\text {os }} 3-4$, p. 259-276.

MÉzIOU, F. (1991), «Areas of strength and weakness in the adoption of the marketing concept by small manufacturing firms », Journal of Small Business Management, octobre, p. 73-77.

Perreault, J.D. (1997), «Le marketing de la PME», GREPME, Les PME: Bilan et perspectives, $2^{\mathrm{e}}$ édition, Paris, Economica, p. 127-149.

RAYMOND, L. et F. BERGERON (1992), « Personal DSS success in small enterprises », Information \& Management, vol. 22, p. 301-308.

RAYMOND, L. et S. BLILI (1997), «Les systèmes d'information », GREPME, Les PME : Bilan et perspectives, $2^{\mathrm{e}}$ édition, Paris, Economica, p. 231-253.

RAYMOND, L., P.-A. JULIEN et C. RAMANGALAHY (2001), « Technological scanning by small Canadian manufacturers », Journal of Small Business Management, vol. 39, $n^{0} 2$ (à paraître).

SALERN, F. (1997), «Innovation, marketing et stratégie », Revue Française du Marketing, $\mathrm{n}^{\circ} 164$, p. $5-30$.

SAMLI, A.C. (1996), Information-driven Marketing Decisions : Development of Strategic Information Systems, Westport, Connecticut, Quorum Books. 
SAPIENZA, H.J., K.G. SMITH et M.J. GANNON (1988), «Using subjective evaluations of organizational performance in small business research », American Journal of Small Business, vol. $12, \mathrm{n}^{\circ} 3$, p. $45-53$.

SAPORTA, B. (1986), Stratégies pour la PME, Paris, Les Éditions Montchrestien.

SERRAF, G. (1977), « Une méthodologie du succès des entreprises de dimension humaine », Revue Française du Marketing, cahier 67, mars-avril, p. 13-48.

THONG, J.Y.L. (1999), « An integrated model of information systems adoption in small business », Journal of Management Information Systems, vol. 15, $\mathrm{n}^{\circ}$ 4, p. 187-214.

TRAYNOR, K. et S.C. TRAYNOR (1989), « Marketing approaches used by high-tech firms », Industrial Marketing Management, vol. 18, p. 281-287.

VARADARAJAN, P.R. et S. JAYACHANDRAN (1999), « Marketing strategy : an assessment of the state of the field and outlook », Journal of the Academy of Marketing Science, vol. $27, \mathrm{n}^{\circ} 2$, p. 120-143.

VENKATRAMAN, N. (1989), « Strategic orientation of business enterprises : the construct, dimensionally and measurement », Management Science, vol. 35, $\mathrm{n}^{\circ}$ 8, p. 942-962.

WREN, B.M., J.T. SiMPSON et C. PAUL (1998), « Marketing channel relationships among small business », International Small Business Journal, vol. 16, nº 4, p. 64-78.

ZINKHAN, G.M., E.A. JOACHIMSTHALER et T.C. KINNEAR (1987), « Individual differences and marketing decision support system usage and satisfaction », Journal of Marketing Research, vol. 24, n 2 , p. 208-214. 